\title{
Desafios na Formação de Professores de Computação: Reflexões e Ações em Construção
}

\author{
Wilk Oliveira $^{1}$, Adão Caron Cambraia ${ }^{2}$ \\ ${ }^{1}$ Instituto de Ciências Matemáticas e de Computação (ICMC) - Universidade de São \\ Paulo (USP) - São Carlos, SP - Brasil \\ ${ }^{2}$ Instituto Federal de Educação, Ciência e Tecnologia Farroupilha (IFFAR) - Santo \\ Augusto - RS - Brasil \\ wilk.oliveira@usp.br, adao.cambraiadiffarroupilha.edu.br
}

\begin{abstract}
One of the main challenges of education in Brazil is the training of teachers for the teaching of Computer Science in Basic Education. In Brazil, the undergraduate course responsible for the training of these teachers is the "Licenciatura em Computação" graduation course. Despite the importance of training these professionals, this training process still faces difficulties. Thus, in this paper, we discuss a series of challenges in this formative process. The study was based on the analysis of the studies published in the $V$ "Licenciatura em Computação" Workshop. As the main contribution, we present a series of challenges associated with directions that can be used to leverage the quality of the course and the overall Computer Science teaching in Brazil.
\end{abstract}

Resumo. Um dos principais desafios da educação no Brasil é a formação de professores para o ensino de Computação na Educação Básica. No brasil, o curso de graduação responsável pela formação desses professores, é o curso de Licenciatura em Computação. Apesar da importância da formação desses profissionais, tal processo de formação ainda passa por dificuldades. Assim, nesse artigo, discutimos uma série de desafios nesse processo formativo a partir das análises dos estudos publicados no V Workshop da Licenciatura em Computação. Como contribuição principal, apresentamos de uma série de desafios associados a direcionamentos que podem ser usados no intuito de alavancar a qualidade do curso e do ensino de Computação no Brasil.

\section{Introdução}

Um dos principais desafios relacionados ao ensino de Computação no Brasil é a formação de professores, que integre conhecimentos computacionais e pedagógicos (França et al. 2012; Santos et al. 2017(a); Torcate et al. 2018). No Brasil, o curso que forma esses profissionais é o curso de Licenciatura em Computação (LC) (SBC, 2017). Apesar da importância do curso e da formação de desses profissionais, tem se destacado a necessidade de identificar e discutir seus desafios, e, a partir disso, traçar estratégias para enfrentá-los (Santos et al. 2017(b); Oliveira et al. 2020).

Diante dessa necessidade, surge o V Workshop da Licenciatura em Computação (WLIC 2019), evento paralelo do Congresso Brasileiro de Informática na Educação (CBIE 2019), cujo principal objetivo foi realizar discussões que venham de encontro 
aos grandes desafios do curso e reunir pesquisadores para discutir ações na LC, por meio de apresentações de trabalhos científicos e palestras, constituindo o que Bachelard (1996) denomina de cidade científica. O evento teve 25 trabalhos submetidos, dos quais 10 (apresentando e discutindo desafios) foram aceitos para publicação e apresentação durante o evento.

A partir do objetivo do referido workshop, surge a necessidade de mapear os principais desafios (alinhados especificamente a formação de professores) apresentados no evento e encaminhar propostas no sentido de enfrentá-los ou proporcionar às comunidades linhas de ação. Assim, o principal objetivo desse texto é destacar os principais desafios elencados nos textos apresentados no WLIC 2019 e construir linhas de reflexão e ação para continuar reforçando a atuação dos cursos de LC nas comunidades locais, bem como, fazer um indicativo do delineamento das próximas temáticas a serem levadas em consideração nos eventos sobre Educação e Computação.

\section{Condução do Estudo}

O design do estudo foi organizado em três etapas, adaptadas a partir do protocolo proposto por Kitchenham e Charters (2007), como sumarizadas na Figura 1: i) análise dos estudos; ii) organização dos tópicos; e iii) descrição dos resultados. Na primeira etapa, os artigos publicados no evento $(\mathrm{N}=10)$ foram analisados através de leitura completa por dois pesquisadores com experiência na área de formação de professores em Computação. Para análise dos textos foi utilizada como método a Análise Textual Discursiva - ATD (Moraes e Galliazzi 2013), por proporcionar elencar reflexões e desafios por meio de categorias. Na segunda etapa, os tópicos foram discutidos pelos pesquisadores e organizados em categorias. Finalmente, na terceira etapa, os resultados foram organizados e descritos de acordo com as categorias identificadas.

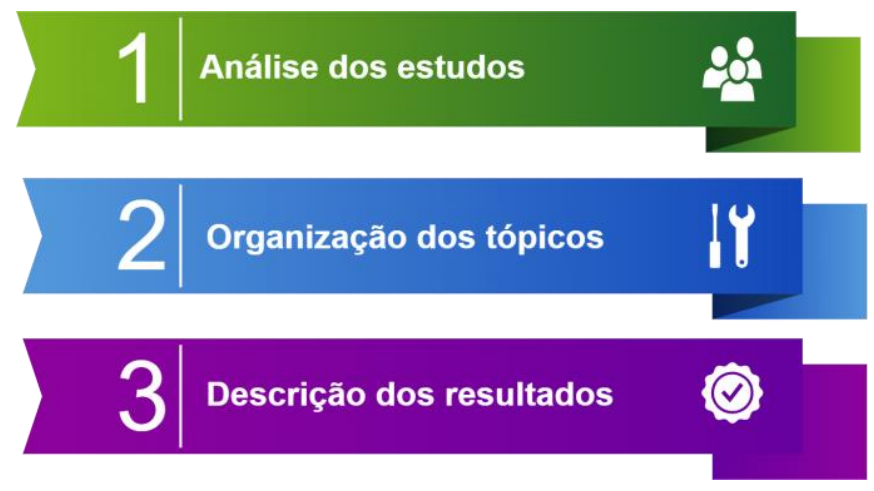

Figura 1. Design do estudo

As categorias foram nomeadas e definidas de acordo com a organização dos anais do evento, que por sua vez, foram definidas de acordo com os tópicos dos artigos. A primeira categoria é formada por textos com as seguintes temáticas: conhecimento profissional; formação continuada; prática profissional; relação licenciatura com a pósgraduação. A segunda categoria é formada por relatos reflexivos sobre o trabalho cotidiano e as principais dificuldades encontradas nas licenciaturas, com as seguintes temáticas: motivação/desmotivação dos licenciados; evasão e equidade de gêneros na LC. 
Escolhemos analisar apenas os artigos do WLIC 2019, em função do evento ter sido construído em caráter especial, unicamente com o objetivo de discutir os desafios do curso, com ações especificas para discussão dos desafios relacionados a formação de professores em Computação. Desse modo, o estudo contribui com a comunidade de pesquisadores e interessados em Educação em Computação e formação de professores para uso de tecnologias, por meio da categorização e discussão dos desafios, facilitando a proposta de reflexões e ações para colocar em movimento a formação de professores de Computação. Os excertos selecionados dos textos do WLIC em análise estão em itálico para diferenciar das citações de autores utilizados na análise. Já nas referências, os textos extraídos do evento estão indicados com $(*)$.

\section{Resultados}

Nessa seção, apresentamos os resultados do estudo. Os resultados foram elencados em duas categorias: "formação de professores em rede" e "a crise das Licenciaturas".

\subsection{Formação de Professores em Rede}

De acordo com Santos et al. (2017a), o curso de Licenciatura em Computação teve a primeira oferta na Universidade de Brasília (UnB) em 1997 e, a segunda, na Universidade de Santa Cruz do Sul (UNISC) em 1999. De acordo com Santos et al. (2017b), nos anos seguintes, houve um crescimento significativo de ofertas e, atualmente, o Ministério de Educação e Cultura - MEC afirma existir mais de 150 cursos no Brasil. De acordo com Cruz et al. (2016), o curso de LC é completo no sentido da formação profissional do professor de computação, pois, o currículo, segundo os autores, integra a própria Computação com a Educação, Matemática, Psicologia, Sociologia, Filosofia, Línguas Portuguesa e Inglesa, demonstrando que sua principal característica é a interdisciplinaridade. Apesar disso, a profissão de professor de Computação não se encontra em solidez; são poucos os espaços legalmente constituídos nas empresas e como professor de Computação na Educação Básica (Santos et al. 2017a; Oliveira et al. 2020).

O desafio da disponibilidade é destaque no texto escrito por Leite et al. (2019) que o apresentam como algo ainda preocupante nas escolas e evidenciam as diferenças entre escolas públicas e privadas em relação a aquisição de infraestrutura tecnológica. Nos estudos que realizam percebem que o laboratório de informática está com "os dias contados", pois, cada vez mais, principalmente nas escolas privadas, ocorre uma substituição por tecnologias móveis (netbooks, notebooks, tablets, entre outros) e como saída, ao desafio da disponibilidade, se preconiza a necessidade de formação de professores para construir metodologias sobre o desenvolvimento do pensamento computacional e para utilização dos recursos que os alunos trazem de casa (smartphones, tablets). Isso é corroborado por Cambraia (2018) ao destacar a importância da Computação Desplugada ${ }^{1}$ e do Bring Your Own Device - BYOD (traga seu próprio dispositivo) como uma forma de superar o desafio da disponibilidade, principalmente nas escolas públicas, seguida de uma formação permanente de professores.

\footnotetext{
${ }^{1}$ A sugestão da Computação Desplugada como alternativa ao desafio da disponibilidade não significa que esse conhecimento seja valorizado apenas diante do desafio. Entende-se que o pensamento computacional por meio da Computação Desplugada seja um conhecimento importante para a Educação Básica assim como a escrita e leitura (WING 2006).
} 
A formação permanente de egressos da LC foi demarcada por Marques et al. (2019), que apontam uma defasagem formativa na medida em que percebem que poucos professores que atuam na Educação Básica distinguem entre pensamento computacional e cultura digital.

\begin{abstract}
"As evidências analisadas neste trabalho apontam que os cursos de $L C$ possuem desafios contundentes no sentido de olhar para seu egresso e repensar a formação oferecida no decorrer do curso e, inclusive, a formação dos professores que compõem o corpo docente do referido curso, questionando-se sobre qual a experiência que possuem no ensino de Pensamento Computacional e Cultura Digital na escola" (MARQUES et al. 2019, p.8).
\end{abstract}

Essa constatação foi possível pela interação do Curso de Licenciatura com escolas de Educação Básica por meio de um projeto de extensão oferecido pelos autores, na cidade de Santa Cruz do Sul (RS - Brasil), para construção de materiais didáticos sobre Computação Desplugada. A referida interação corrobora o que Cambraia (2019) destaca como umas das características cruciais para o desenvolvimento profissional docente, que é a interação Licenciatura e Educação Básica por meio da indissociabilidade entre Ensino, Pesquisa e Extensão, proporcionando um maior diálogo entre professores formadores, licenciandos e professores da Educação Básica na construção de projetos integradores. Também é importante destacar que a aproximação por meio de disciplinas como Metodologias, Práticas como Componente Curricular e Estágios não significa que ocorra interação de professores da Educação Básica com professores formadores e licenciandos. Em muitos casos, essas aproximações não provocam interações e pesquisas colaborativas.

Além da interação da Licenciatura com a Educação Básica, Oliveira et al. (2019) estudaram sobre a interlocução da Licenciatura em Computação com um Mestrado Profissional, em que os licenciandos oferecem uma oficina de robótica para os mestrandos. As contribuições ao curso de Licenciatura em Computação foram

[...] "que durante os processos de planejamento e aplicação da oficina,
puderam desenvolver habilidades e competências necessárias a suas futuras
práticas docentes, tais como: a definição de objetivos educacionais, o
processo de adequação de materiais didáticos, o ensino de programação, a
integração de conteúdos curriculares às TICs" (OLIVEIRA et al. 2019, p.
10).

A elaboração dos conhecimentos profissionais de professores como: definir objetivos, construção de materiais didáticos, integração de conteúdos disciplinares são conhecimentos profissionais corroborados por Cambraia e Biondo (2019), que além desses, pautados em Carr e Kemmis (1988), trouxeram indícios da necessidade em desenvolver processos de pesquisa-ação com enfoque crítico e emancipatório na formação de professores para romper com a desarticulação entre conhecimentos pedagógicos e da computação e proporcionar a constituição dos conhecimentos didáticos do conteúdo. As principais características da formação em rede são:

\footnotetext{
"Interação dos cursos de Formação de Professores com Escola de Educação Básica com interlocução entre professores formadores, professores e alunos da Educação Básica e licenciandos. - Compreensão do conhecimento do conteúdo com profundidade para desenvolver metodologias criativas $e$ interdisciplinares. - Priorizar várias formas de planejamento, dentre os quais, o planejamento colaborativo como um processo reflexivo para criar
} 
desafios que envolvam os alunos e alcançar objetivos. - Transformar conhecimentos científicos em conhecimentos escolares. - Produção de materiais didáticos levando em conta as características dos alunos. - Escrita reflexiva para produzir conhecimentos cientificos" (CAMBRAIA, BIONDO 2019, p. 9).

Em resumo, as características elencadas sobre os espaços formativos proporcionaram a constituição do conhecimento didático do conteúdo no coletivo de professores que participaram da pesquisa-ação, mas não significa que essas características são suficientes. A formação de professores é complexa e, por isso, os conhecimentos profissionais são permanentemente reconstruídos e ocasionam uma reinvenção da profissão. Ao constatar os desafios reconhece-se a profissão docente em construção, inacabada. A espiral da pesquisa-ação, que envolve o planejamento, ação, observação e reflexão proporciona a pesquisa permanente na formação docente, pois a computação e educação se transformam permanentemente, exigindo a constituição de um professor-pesquisador. Assim, a formação de professores em rede por meio de uma pesquisa-ação crítica e emancipatória é um caminho em construção que precisa ser expandido entre os cursos de LC do Brasil, proporcionando formações que articulem teoria e prática.

\subsection{Crise das Licenciaturas}

Nessa categoria os estudos selecionados tratam de desafios a serem enfrentados no cotidiano dos cursos analisados, como por exemplo: falta de incentivo político à docência no ensino básico e a importância de diferentes metodologias nas disciplinas dos cursos para evitar a evasão dos alunos.

É sabido que os primeiros momentos dos estudantes em um determinado curso são cruciais para continuidade dos estudos. Nesse sentido, Krzyzanowski et al. (2019) em seu estudo realizou uma busca pelas informações e projetos pedagógicos de LC no Brasil, onde analisou as disciplinas introdutórias de dois cursos de uma universidade do sul do Brasil (a saber Universidade Federal do Paraná (UFPR)), analisando os índices de reprovação e evasão dos alunos nas disciplinas introdutórias e sua ligação com a evasão.

No curso analisado, os autores identificaram que "em média, $62,5 \%$ dos alunos que reprovaram na primeira disciplina de Algoritmos e Programação por nota e 70\% dos que reprovaram por frequência acabaram trancando o curso" (KRZYZANOWSKI et al. 2019). Ao mesmo tempo, "em média, metade dos alunos que conseguem aprovação nessa disciplina mantêm-se matriculados no curso" (KRZYZANOWSKI et al. 2019). Tais resultados confirmam que possivelmente as disciplinas introdutórias são de grande importância na permanência dos estudantes, bem como, destaca a importância de se propor e avaliar outras metodologias para o ensino dessas disciplinas.

Pontes et al. (2019) apresentou em seu estudo desafios e possibilidades vivenciadas por licenciandas indígenas (declaradas do sexo feminino) em Computação na busca de incentivar meninas na tecnologia. O trabalho de Pontes et al. (2019) destaca desafios com base na investigação do contexto das alunas de uma escola indígena de uma cidade de um estado do Nordeste brasileiro (a saber, Paraíba - PB), destacam-se como principais problemas "a falta de perspectiva de continuidade do ensino; baixos recursos da escola e comunidade na qual estão inseridas; e a falta de iniciativas de apoio na escola para incentivo tecnológico e de conhecimento da área de Computação" (PONTES et al. 2019). Nota-se que assim como em outros cursos da área de Tecnologia 
da Informação e Comunicação, existe uma disparidade entre estudantes do sexo masculino e feminino, sendo mais comum a participação de estudantes do sexo masculino. Os resultados do estudo de Pontes et al. (2019) confirmam que problemas relacionados a Educação Básica, baixos recursos e falta de incentivo do poder público são causas importantes nessa problemática. Mas, uma análise mais aprofundada exige estudos para entender os fatores que mantêm as mulheres a margem do processo de produção tecnológica e científica, mesmo quando são as principais mentoras (PLANT 1999).

Nesta mesma linha, Gomes et al. (2019) realizou uma pesquisa com estudantes de LC e Bacharel em Sistemas de Informação (BSI) de uma universidade do nordeste do Brasil (UFPB - Universidade Federal da Paraíba), objetivando aferir o nível de informação sobre as subáreas de computação, bem como, os níveis de interesse em relação a essas subáreas. O percentual de discentes foi de 12,7\% de estudantes declaradas do sexo feminino no curso de Licenciatura em Computação e 10,5\% no Bacharel em Sistemas de Informação (GOMES et al. 2019). Como um dos resultados, notou-se que a subárea Informática na Educação se encontra com 42,9\% de interesse.

De acordo com os autores do texto, com relação a motivação para a escolha de tais subáreas, houve equivalências entre as motivações, onde $14 \%$ se mantiveram imparciais, ou seja, considerando tanto as condições internas quanto externas, das demais $36 \%$ são motivadas por fatores internos e do valor restante $(50 \%)$, são motivadas por condições externas guiadas dentro da universidade através de professores, disciplinas, palestras, etc. (GOMES et al. 2019). Os resultados obtidos por Gomes et al. (2019) demostram que o contexto no qual as discentes estão inseridas é de fundamental importância no desenvolvimento das estudantes ao longo do curso.

Nascimento et al. (2019) buscaram compreender a influência de fatores internos à formação do educador em Computação de uma universidade do Nordeste brasileiro (a saber Universidade Federal da Paraíba - UFPB). Os resultados iniciais (como destacado pelos autores) mostram que maioria dos estudantes (de ambos os cursos) sabem a diferença entre o bacharelado e a licenciatura em Computação (NASCIMENTO et al. 2019). Esse resultado mostra uma evolução neste cenário, dado que em pesquisas anteriores na mesma instituição mostravam que a maioria dos alunos não sabia essa diferença. Segundo os autores, por outro lado, quando perguntados se queriam seguir a carreira de professor, $80 \%$ deles responderam que não pretendiam seguir a carreira de magistério.

O estudo de Nascimento et al. (2019) elencou ainda os principais motivos para esse desejo dos alunos de não atuar como professor, sendo eles: falta de vocação; medo de atuar como professor; baixa estima e falta de Computação nos currículos da educação básica os motivos para não querer ser professor. Essas constatações nos trazem indícios de como a formação precisa complexificar as discussões sobre a qualidade da educação, pois a computação tem muito a oferecer não como uma disciplina estanque, mas como um conhecimento interdisciplinar importante para inovar na educação.

Além disso, a desvalorização, tanto financeira quanto profissional (o valor que a sociedade atribui ao professor), foi mencionada (NASCIMENTO et al. 2019). Assim como, a falta de incentivo por parte de seus professores durante o Ensino Médio foi notada. Tal resultado coloca em destaque a falta de valorização do professor e, por 
conseguinte, a desmotivação por parte dos jovens em seguir a carreira, o que representa que futuramente corre-se o risco da falta de professores em todas as áreas e, principalmente, na computação que é uma área em franco crescimento.

Gonçalves e Scaico (2019) conduziram um estudo com professores (também da UFPB) no intuito de identificar como os professores têm lidado com novas práticas de ensino (i.e., aprendizagem ativa). O estudo de Gonçalves e Scaico (2019) obteve a participação de dezessete professores do curso de LC, segundo os autores, permitindo o levantamento de dados sobre 29 disciplinas, correspondente a $59 \%$ do corpo docente e a $67 \%$ das disciplinas do curso (GONÇALVES \& SCAICO 2019). A maioria dos professores participantes do estudo foram bacharéis na área de tecnologia ou engenharia, portanto, a minoria possui formação pedagógica "de base" em outras áreas do conhecimento (GONÇALVES \& SCAICO 2019), mas não possuem formação pedagógica.

Dentre os principais resultados, os pesquisadores destacam que "a maioria dos docentes afirmaram utilizar aprendizagem ativa nas suas aulas, tais como: gamificação, aprendizagem por pares, aprendizagem baseada em projeto, sala de aula invertida e PBL" (GONÇALVES \& SCAICO 2019). Ainda, destacam que entre os professores que dizem não saber do que se trata aprendizagem ativa ou não sabem definir aprendizagem ativa, a maioria aplica técnicas de aprendizagem ativa mesmo sem saber que está aplicando a técnica. Se por um lado os resultados de Gonçalves e Scaico (2019) demostram que os professores têm conseguido aplicar essas técnicas, por outro lado, mostra que o assunto tem sido pouco discutido entre os docentes, e, por conseguinte, entre os discentes, se fazendo necessária formação continuada de professores para suprir ou minimizar os efeitos da falta de formação de base em Pedagogia.

Na categoria "crise das licenciaturas" emergiram muitos problemas na formação inicial de professores e essas problemáticas são em decorrência da falta de valorização da profissão docente. Ou seja, os desafios agrupados nessa categoria destacam que a LC passa por um momento de dificuldade inerente a crise da profissão docente. Para uma modificação desse quadro momentos como o WLIC são importantes para reforçar a atuação dos futuros professores junto às comunidades locais, reforçando e qualificando a presença do ensino da computação. Com a concretização de encontros e estudos oportunizados, como no WLIC é possível notar que os professores, estudantes e comunidade em geral têm compreendido os objetivos do curso e a continuidade desses estudos pode ampliar a contribuição da LC para a Educação Básica. A constatação dos desafios é como colocar um "espelho" para refletir nossa imagem, proporcionando aos atuais e futuros professores possibilidades de agir diante das dificuldades.

\section{Discussões para continuidade do ciclo formativo}

Dentre os desafios foram elencados os que mais receberam destaque nos textos analisados para proporcionar o desenvolvimento de um olhar crítico e reflexivo sobre o fazer docente. Diante das análises anteriormente apresentadas, foi possível chegar a uma síntese 10 desafios, ações e reflexões para possibilitar a transformação dos cursos de formação de professores de computação e impulsionar novas pesquisas sobre a formação de professores: a) reestruturação didático/pedagógica de disciplinas introdutórias para evitar a evasão; b) ações de integração e expansão de cursos de computação, aumentando a divulgação de ações de impacto; $c$ ) fortalecimento político 
por meio de ações da Sociedade Brasileira de Computação para destacar a importância do professor de computação na Educação Básica e valorização financeira da carreira docente; d) divulgação das propostas e ações realizadas pela LC na sociedade, que representem uma concretização da produção do conhecimento; e) desenvolvimento de ações que promovam a equidade de gênero nos cursos de LC; f) desencadear formação em rede para produzir conhecimento profissional docente, integrando conhecimentos da Computação e Pedagógicos; g) ampliar eventos para potencializar a pesquisa na formação de professores de computação; $h$ ) planejamento colaborativo dos projetos integradores entre licenciandos, professores formadores com professores e alunos da escola de Educação Básica; i) promover a indissociabilidade no Ensino, Pesquisa e Extensão; $j$ ) ampliar e qualificar as publicações da área.

Para próximas ações, sugerimos a apresentação dessa sistematização, potencializando aprendizagens e transformações na formação de professores de computação, constituindo e ampliando redes de pesquisadores em Educação e Computação, bem como, a necessidade de estudos que analisem as ações propostas e privilegiem a socialização de boas práticas na formação de professores e, principalmente, ações que concretizem o ensino de computação na Educação Básica, trazendo indícios da importância de um novo profissional no espaço da escola.

\subsection{Limitações}

O estudo possui algumas limitações que são inerentes ao próprio caráter da pesquisa e que devem ser consideradas na interpretação dos resultados e em possíveis avanços do estudo. Inicialmente, é possível que as leituras não sejam fidedignas ao que foi apresentado nos estudos publicados nos anais do evento, pois a reconstrução de textos unitarizados é um exercício de autoria com novas interpretações para construção do metatexto, características da ATD. Diante disso, sugerimos que os textos publicados no evento possam ser lidos como fonte complementar aos resultados apresentados nesse artigo. Os textos apresentados no evento versaram sobre diferentes aspectos que podem não ter sido abordados nesse artigo. Assim, sugerimos que novos desafios e discussões possam ser abordadas em estudos futuros. Finalmente, os desafios apresentados nesse artigo não pretendem cobrir todos os desafios do curso e da formação de professores de Computação, mas sim, promover uma discussão contemporânea baseada nos resultados de pesquisas recentes na área. Tal objetivo é corroborado pela escolha de analisarmos artigos provenientes de um evento com foco principal da apresentação de desafios do curso de LC.

\section{Considerações Finais}

Um dos principais desafios na educação Brasileira é a formação de professores para o ensino de Computação na educação básica. Diante disso, nesse artigo apresentamos uma série de desafio relacionados a formação desses profissionais a partir de discussões realizadas dentro do curso responsável por formar tais profissionais. Os resultados apontam especificamente para a formação de professores em rede e a crise das licenciaturas como desafios do curso e da formação desses profissionais. Apresentamos também uma série de propostas para enfretamento desses desafios. Esperamos como ações futuras, a realização de novas ações que possam fortalecer o curso de LC e a formação de professores de computação para educação básica no Brasil. 
IX Congresso Brasileiro de Informática na Educação (CBIE 2020)

Anais do XXVI Workshop de Informática na Escola (WIE 2020)

\section{Referências}

Bachelard, G. (1996). A formação do espírito científico. Rio de janeiro: Contraponto.

Cambraia, A. C. (2018). Aprender e ensinar na Cibercultura: Desafios e Perspectivas Inovadoras. In: V Seminário de Inclusão Digital, 2018, Passo Fundo. Cultura Digital na Educação. ISSN: 2238-5916. Passo Fundo: Ed Universidade de Passo Fundo, v. 5. p. 1-10.

Cambraia, A., \& Biondo, U. R. (2019). Construção do Conhecimento Profissional do Professor de Computação pela pesquisa-ação. In Anais dos Workshops do Congresso Brasileiro de Informática na Educação (Vol. 8, No. 1, p. 1). *

Carr, W.; Kemmis, S. Teoria crítica de la enseñanza: investigación-acción en la formación del profesorado. Barcelona: Martinez Roca, 1988.

Cruz, M. K., Becker, F., \& Hinterholz, L. (2016). Carga Horária Prática na Formação de Professores de Computação e Informática Educativa. In Anais do Workshop de Informática na Escola (Vol. 22, No. 1, p. 698).

Falcade, A., Duarte, A. V., Richter, C., dos Santos, J. N., Dornelles, L., \& Barbosa, S. (2019). Prática enquanto componente curricular: um relato de experiência. In Anais dos Workshops do Congresso Brasileiro de Informática na Educação (Vol. 8, No. 1, p. 11). *

França, R. D., Silva, W. D., \& Amaral, H. D. (2012). Ensino de ciência da computação na educaçao básica: Experiências, desafios e possibilidades. In $X X$ Workshop sobre Educação em Computação (p. 4).

França, R. S., \& do Amaral, H. J. C. (2013). Ensino de computação na educação básica no brasil: Um mapeamento sistemático. In XXI Workshop sobre Educação em Computação.

Gonçalves, A., \& Scaico, P. (2019). O Desafio de Professores da Licenciatura em Computação ao Usarem Práticas de Aprendizagem Ativa: Um Estudo de Caso na Universidade Federal da Paraíba. In Anais dos Workshops do Congresso Brasileiro de Informática na Educação (Vol. 8, No. 1, p. 91). *

Kitchenham, B., \& Charters, S. (2007). Guidelines for performing systematic literature reviews in software engineering.

Krzyzanowski, L., Beleti Jr, C., Santiago Jr, R., \& Tostes, R. A. (2019). Ensino de programação: um estudo preliminar nos cursos de licenciatura em Computação no Brasil. In Anais dos Workshops do Congresso Brasileiro de Informática na Educação (Vol. 8, No. 1, p. 21). *

Leite, M., Reinaldo, F., \& Oliveira, Q. (2019). Ações de intervenção na formação de professores com base em metodologia tecnológica. In Anais dos Workshops do Congresso Brasileiro de Informática na Educação (Vol. 8, No. 1, p. 61). *

Marques, S., da Cruz, M. K., \& Schulz, F. (2019). Formação Continuada de Licenciados em Computação para Trabalho com Computação na Escola. In Anais dos Workshops do Congresso Brasileiro de Informática na Educação (Vol. 8, No. 1, p. 31). *

Melo, I., Rocha, N., \& Scaico, P. (2019). Eles não querem ser professores. In Anais dos Workshops do Congresso Brasileiro de Informática na Educação (Vol. 8, No. 1, p. 51). *

Mioto, F., Petri, G., von Wangenheim, C. G., Borgatto, A. F., \& Pacheco, L. H. (2019). bASES21-Um Modelo para a Autoavaliação de Habilidades do Século XXI no Contexto do Ensino de Computação na Educação Básica. Revista Brasileira de Informática na Educação, 27(1). 
Moraes, R., Galiazzi, M. D. C., \& Ramos, M. G. (2013). Aprendentes do aprender: um exercício de análise textual discursiva. In Escola de Química e Alimentos.

Oliveira, A., Nascimento, L. T., \& Macedo, D. (2019). Licenciatura em Computação: Um Relato de Experiência Utilizando Robótica na Formação de Professores. In Anais dos Workshops do Congresso Brasileiro de Informática na Educação (Vol. 8, No. 1, p. 71). *

Oliveira, W., França, R., Lemos, A., da Cruz, M. K., Scaico, P., Amaral, H., Teixeira, L. P. (2020). Os Desafios Enfrentados pela Licenciatura em Computação que a Comunidade de Educação em Computação Precisa Conhecer. In XXVIII Workshop sobre Educação em Computação (WEI).

Plant, S. (1999) Mulher digital: feminismo e as novas tecnologias. Traduzido por: Ruy Jungmann. Rio de Janeiro: Record: Rosa dos Tempos.

Pontes, M. M. G., Silva, W. P., Dantas, V. F., \& de Lima Costa, T. K. (2019). Desafios no incentivo de meninas para a área de Computação. In Anais dos Workshops do Congresso Brasileiro de Informática na Educação (Vol. 8, No. 1, p. 41). *

Santos Gomes, V. H., de Araújo, I., \& Soares, I. (2019). Percepções das Discentes dos Cursos de TI Sobre as Subáreas da Computação. In Anais dos Workshops do Congresso Brasileiro de Informática na Educação (Vol. 8, No. 1, p. 81). *

Santos, W. O., Hinterholz, L., \& Silva, C. (2017a). Licenciatura em Computação: Desafios e oportunidades na perspectiva do professor. In Anais do Workshop de Informática na Escola (Vol. 23, No. 1, p. 705).

Santos, W. O., Silva, C., \& Hinterholz, L. (2017b). Licenciatura em Computação: Desafios e oportunidades na perspectiva do estudante. In Anais do Workshop de Informática na Escola (Vol. 23, No. 1, p. 885).

Silva, J. A. S., Cordeiro, H. V. F., da Silva, S. R. F., \& Gomes, A. L. (2018). Estágio Docente de Licenciatura de Computação: Um Ensaio do Ensino de Computação no Ensino Fundamental. In $26^{\circ}$ Workshop sobre Educação em Computação (WEI 2018) (Vol. 26, No. 1/2018). SBC.

Silveira, S. R., Pereira, A. S., Bertolini, C., Parreira, F., \& Cunha, G. (2018). Potencialidades e Desafios do Curso de Licenciatura em Computação a Distância da UFSM/UAB. In Anais dos Workshops do Congresso Brasileiro de Informática na Educação (Vol. 7, No. 1, p. 1061).

Sociedade Brasileira de Computação (SBC) (2017), "Referenciais de Formação em Computação: Educação Básica", http://www.sbc.org.br/documentos-da$\mathrm{sbc/send/131-curriculos-de-referencia/1166-referenciais-de-formacao-em-}$ computacao-educacao-basica-julho-2017, March.

Torcate, A. S., de Farias, M. U. F., da Silva, S. R. F., \& Martins, C. S. (2018). A inserção de Computação como disciplina no Ensino Fundamental: Desafios e Conquistas em Estágio Supervisionado. In $26^{\circ}$ Workshop sobre Educação em Computação (WEI 2018) (Vol. 26, No. 1/2018). SBC.

Wing, J. M. (2006). Computational thinking. In Commnications of the ACM, pages 3335 . 